\title{
Application of electric pulsed power on fruit body production of edible and medicinal mushrooms
}

\author{
Shoji Ohga ${ }^{1}$ * \\ ${ }^{1}$ Division of Forest Environmental Sciences, Department of Agro-environmental Sciences, Faculty of Agriculture, Kyushu University, Sasaguri, \\ Fukuoka 811-2415, Japan
}

Received on 13 November 2012, revised on 17 December 2012, accepted on 18 December 2012

\begin{abstract}
Effect of pulsed power was investigated on fruit body formation of 10 edible mushrooms, Lentinula edodes, Glifola frondosa, Pholiota nameko, Flammulina velutipes, Hypsizygus marmoreus, Pleurotus ostreatus, Pleurotus eryngii, Pleurotus abalonus, Agrocybe cylindracea and Sparassis crispa. Pulsed power of 100-170 kV was directly charged to the substrate just before fructification. The effect of the pulsed power resulted to promote for 10 edible mushrooms fructification. The treatment especially stimulated the fructification on Pleurotus species.
\end{abstract}

Key words : Electric pulsed power, Mushroom, Fruit body

\section{Introduction}

Cultivation of edible mushrooms on sawdust-based substrate is steadily improving to effective methods. Especially, efficient techniques are useful for this cultivation method. Biological efficiency has been improved by optimizing various factors, such as substrate formula, strain type, culture maturity, water condition and other environmental conditions of the cultivation room.

Electric power utility is now applied to various agricultural crops, especially in horticultural fields. Cultivation system has been improved with electricity utilization in the field of systematic house crops such as tomato, lettuce, strawberry and various kinds of flower. Effect of air ions on plants have experimented. Fruit body production was promoted by the electric impulse treatment on the logwood or sawdust substrate (Ohga et al, 2001) of L. edodes. Effect of the pulsed power was investigated on the fruit body formation in the sawdust-based substrate of 10 edible mushrooms.

\footnotetext{
*Corresponding author: Tel: +81-92-948-3116

E-mail address: ohga@forest.kyushu-u.ac.jp
}

\section{Materials and Methods}

\section{Strains}

Ten edible mushroom species used in this study, Lentinula edodes (KUMB 114), Grifora frondosa (KUMB 124), Pholiota nameko (KSMK 45), Flammulina velutipes (KS 72), Hypsizygus marmoreus (KUMB 111), Pleurotus ostreatus (KSMK 39), Pleurotus eryngii (KUMB 117), Pleurotus abalonus (KSMK 43), Agrocybe cylindracea (KS 67) and Sparassis crispa (KUMB 1201). All of these strains were stocked in Kyushu university fungi collection, and maintained on a potato dextrose agar medium (Difco) at $4{ }^{\circ} \mathrm{C}$.

\section{Culture media and Growth conditions}

Fruit body production was tested on a plastic bag or bottle (Table 1$)$. The plastic bottle $(800 \mathrm{~mL})$ contained $500 \mathrm{~g}$ substrate. L. edodes and G. frondosa was cultivated on the large bag filled with $2.5 \mathrm{~kg}$ substrate, and $P$. nameko was cultivated on the bottle filled with 700 g substrate. The medium was sterilized by autoclaving at $120^{\circ} \mathrm{C}$ for $30 \mathrm{~min}$ and then allowed to cool to room 
Table 1. The species of 10 edible mushrooms and conditions for cultivations.

\begin{tabular}{lllcc}
\hline \multicolumn{1}{c}{ Mushroom species } & \multicolumn{1}{c}{ Sawdust species } & $\begin{array}{c}\text { Culture vessel and } \\
\text { weight of substrate }\end{array}$ & $\begin{array}{c}\text { Period for spawn run } \\
\text { and temperature }\end{array}$ & $\begin{array}{c}\text { Days for fructification } \\
\text { and temperature }\end{array}$ \\
\hline $\begin{array}{l}\text { Lentinula edodes } \\
\text { Grifola frondosa }\end{array}$ & Fagus crenata & Bag $(2.5 \mathrm{~kg})$ & 60 days, $22^{\circ} \mathrm{C}$ & 20 days, $15^{\circ} \mathrm{C}$ \\
Pholiota nameko & Fagus crenata & Bag $(2.5 \mathrm{~kg})$ & 40 days, $22^{\circ} \mathrm{C}$ & 20 days, $15^{\circ} \mathrm{C}$ \\
Flammulina velutipes & Cryptomeria japonica & Bottle $(700 \mathrm{~g})^{\mathrm{b})}$ & 80 days, $22^{\circ} \mathrm{C}$ & 20 days, $15^{\circ} \mathrm{C}$ \\
Hypsizygus marmoreus & Fagus crenata & Bottle $(500 \mathrm{~g})$ & 30 days, $15^{\circ} \mathrm{C}$ & 30 days, $\left.7^{\circ} \mathrm{C}\right)$ \\
Pleurotus ostreatus & Cryptomeria japonica & Bottle $(500 \mathrm{~g})$ & 80 days, $22^{\circ} \mathrm{C}$ & 20 days, $15^{\circ} \mathrm{C}$ \\
Pleurotus eryngii & Cryptomeria japonica & Bottle $(500 \mathrm{~g})$ & 30 days, $22^{\circ} \mathrm{C}$ & 10 days, $15^{\circ} \mathrm{C}$ \\
Pleurotus abalonus & Cryptomeria japonica & Bag $(2.5 \mathrm{~kg})$ & 30 days, $22^{\circ} \mathrm{C}$ & 20 days, $15^{\circ} \mathrm{C}$ \\
Agrocybe cylindracea & Cryptomeria japonica & Bottle $(500 \mathrm{~g})$ & 30 days, $22^{\circ} \mathrm{C}$ & 20 days, $15^{\circ} \mathrm{C}$ \\
Sparassis crispa & Larix leptolepis & Bottle $(500 \mathrm{~g})$ & 30 days, $22^{\circ} \mathrm{C}$ & 10 days, $15^{\circ} \mathrm{C}$ \\
\hline
\end{tabular}

a) F. velutipes, H. marmoreus, P. ostreatus, P. eryngii, P. abalonus and A. cylindracea were adopted the kinkaki treatment just before the transferring of cultures into low temperature rooms, but other mushrooms were induced without the treatment.

b) The culture bottles were $850 \mathrm{~mL}$ capacity, and a large diameter mouth was used for $P$. nameko cultures.

${ }^{c}$ ) F. velutipes was allowed to incubate at $5^{\circ} \mathrm{C}$ for another 10 days preceding the fruit body development process to produce uniform fruiting initials.

temperature. The sawdust spawn was inoculated at the surface of the plastic bag and bottle substrates, respectively.

All substrates were cultured in the dark during the early phase of fungal growth until 14 days from inoculation, and then were exposed to 500 lux intensity of $12 \mathrm{hr}$ intervals of cool-white fluorescent illumination. Incubation days for mycelial growth and fruiting treatment were specific for various mushrooms. The plastic bag and bottle cultures were continuing incubated longer to continue vegetative mycelial growth. The fructification stimulation, kinkaki treatment (removal of both spawn and the uppermost layer of the medium) was done with the fully matured cultures of $F$. velutipes, $H$. marmoreus, $P$. ostreatus, $P$. eryngii, $P$. abalonus and $A$. cylindracea. Chilling treatment (shift down of temperature) was given to all species cultures after various incubation days. Fruit body production was measured under the low temperature conditions for various days.

\section{Pulsed power treatment}

Pulsed power treatments were done just before the fruiting stages. Pulsed power of 100-170 kV (1.0 kWS) was directly shot through the electrodes from the instrument (Yushin Tech. Co. Ltd) to the mature sawdust-based substrates (Fig. 1).

\section{Results and Discussion}

The flush spontaneously occurred at 5 to 15 days after the pulsed power treatment. Pulsed power resulted to more production of fruit-body in all tested strains (Fig. 2). Difference between the pulsed power and control was already recognized in the primodia formation stage. Number of primodia on the treated substrate was more than that of control. Shapes of pilei and stipe of the fruit body were normal in the treated substrates.

Pulsed power resulted to more production of fruit body in all tested mushrooms. Fructification occurred at 5 to 10 days after the pulsed power treatment. Difference between the pulsed power treatment and control was already recognized in the primodia formation stage. Number of primodia on the treated substrate was more than that of control. Shapes of pilei and stipe of the fruit body were normal in the 


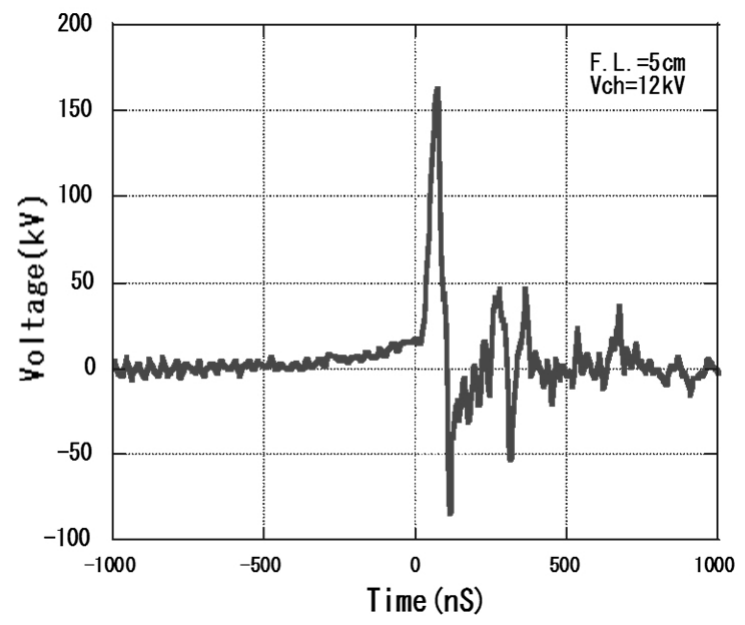

Fig. 1. Shape of electric pulse power.

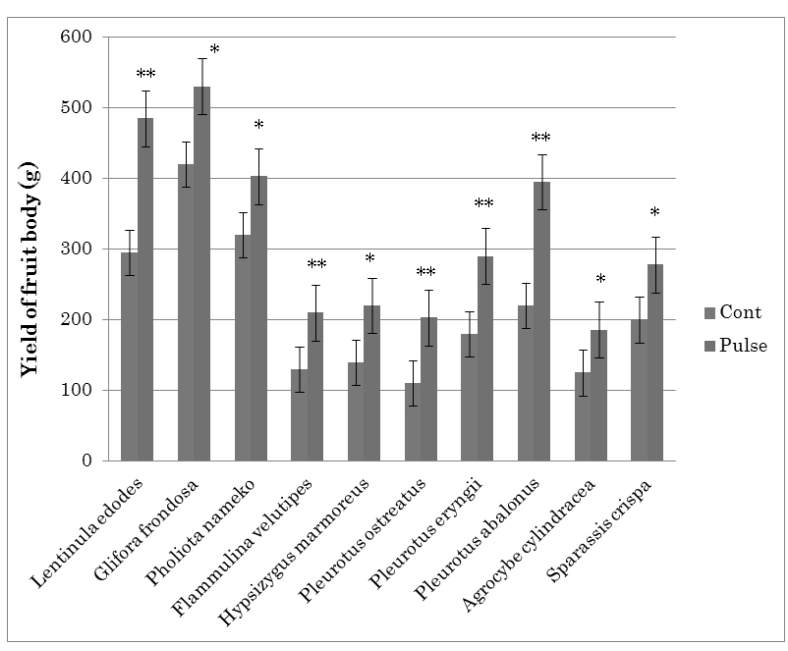

Fig. 2. Effect of the pulsed power stimulation for 10 species mushrooms.

treated substrates.

Pleurotus ostreatus was most sensitive for the pulsed power stimulation. Growth ratio (yield on treated substrate / yield on control) indicated $185 \%$ by the pulsed power treatment. The value was $180 \%$ in $P$. abalonus, and $161 \%$ in $P$. eryngii. In general, the treatment may be effective to Pleurotus $s p$. judging for the significant differences at $\mathrm{p}<0.01$. Also $L$. edodes and $F$. velutipes are excellent with the pulsed power treatment, $164 \%$ and $162 \%$, respectively. Growth ration of $H$. marmoreus and $A$. cylindracea were $157 \%$ and 149\%, respectively. These 2 mushrooms revealed significant differences at $\mathrm{p}<0.05$. There was significant difference on the substrate of $G$. frondosa and

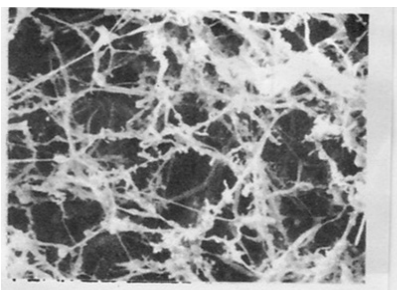

Pulsed power treatment

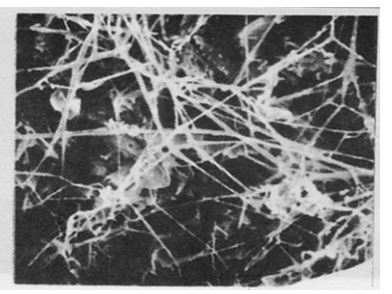

Control
Fig. 3. Morphological changes with pulsed power stimulation on mycelia of Lentinula edodes with scanning electron microscope observation $(\times 500)$.

\section{$P$. nameko yield increase by the treatment.}

The pulsed power treatment also fine resulted to the fructification of $S$. crispa (medicinal mushroom). This mushroom is recently reported for quite high content of B1,3-glucan, and contributed to cancer disease. Fruit body yield increased 130-180\% under pulsed power stimulation compared with control.

Difference for the pulsed power treatment was recognized according to fruit body fructification. Single fruiting type was more sensitive for the pulsed power stimulation than gather fruiting type.

We have also reported the possibility of enhancement of fruit body formation of the mycorrhizal fungi (Laccaria laccata) with pulsed power in nursery and plantations (Ohga and Iida, 2001).

The reason of positive effect of pulsed power for fructification is realized for mycelia stimulation. The cracking phenomenon of mycelia was observed by the pulsed power treatment on the substrate of $L$. edodes. The cracking phenomenon resulted mycelia density incensement (Fig. 3). The cracking was concluded the main effect of fructification promotion of $L$. edodes. Pulsed power stimulation was obviously effective to the yield of some edible mushrooms tested here. Various factors have to examine for the positive effect of these edible mushrooms fructification.

Pulsed power treatment has a possibility to apply the large scale industrial production site. Some application tools for convenient pulsed power supply on the substrate will be also improved in the field of technology. 


\section{References}

Ohga S, Iida S, Koo CD, Cho NS. 2001. Effect of electric impulse on fruit body production of Lentinula edodes in the sawdust-based substrate. Mushroom Science and Biotechnology 9:7-12.

Ohga S. Iida S. 2001. Effect of electric impulse on sporocarp formation of ectomycorrhizal fungus Laccaria laccata in Japanese red pine plantation. Journal of Forest Research 6: 37-41. 